\title{
Ann Arbor Stage III Childhood Lymphoblastic Lymphoma
}

National Cancer Institute

\section{Source}

National Cancer Institute. Ann Arbor Stage III Childhood Lymphoblastic Lymphoma. NCI Thesaurus. Code C9058.

Stage III: T umors or involved lymph node areas occur on both sides of the diaphragm. Stage III also includes any primary intrathoracic (mediastinal, pleural, or thymic) disease, extensive primary intra-abdominal disease, or any paraspinal or epidural tumors (from PDQ). 